OUTP-97-31-P

hep-ph/9707401

\title{
Unification predictions
}

\author{
Dumitru Ghilencea*巴, Marco Lanzagorta ${ }^{\dagger}$, Graham G. Ross*际 \\ *Department of Physics, Theoretical Physics, University of Oxford \\ 1 Keble Road, Oxford OX1 3NP, United Kingdom \\ $\dagger$ High Energy Section, International Centre for Theoretical Physics \\ PO Box 586, Trieste, Italy
}

Published in Nuclear Physics B 511(1,2), 1998, 3-24

\begin{abstract}
The unification of gauge couplings suggests that there is an underlying (supersymmetric) unification of the strong, electromagnetic and weak interactions. The prediction of the unification scale may be the first quantitative indication that this unification may extend to unification with gravity. We make a precise determination of these predictions for a class of models which extend the multiplet structure of the Minimal Supersymmetric Standard Model to include the heavy states expected in many Grand Unified and/or superstring theories. We show that there is a strong cancellation between the 2-loop and threshold effects. As a result the net effect is smaller than previously thought, giving a small increase in both the unification scale and the value of the strong coupling at low energies.
\end{abstract}

\footnotetext{
${ }^{1}$ E-mail address: D.Ghilencea1@physics.oxford.ac.uk

${ }^{2}$ E-mail address: unisol@ictp.trieste.it

${ }^{3}$ E-mail: Ross@thphys.oxford.ac.uk
} 


\section{Introduction}

While the Standard Model continues to provide a remarkably consistent description of essentially all observed phenomena, many physicists think that there must be a more fundamental theory capable of determining many of its features and parameters. To date, the only quantitatively successful predictions for physics beyond the Standard Model are the "unification" predictions for its gauge and Yukawa couplings and for this reason a great deal of attention has been paid to them. At best, the present success of these predictions gives some circumstantial evidence for a stage of supersymmetric unification. As the measurement of the couplings of the Standard Model improves, it is possible to test such unification predictions with ever higher precision and the hope is this will strengthen the case for some underlying unification of the strong, electromagnetic and weak interactions and shed light on its specific form. Furthermore, the scale of unification may provide us with the first indication of unification with gravity because it is predicted in string theories with a value close to that needed for unification. It is the purpose of this paper to test these predictions in detail for a class of models which allow for the addition of heavy states as expected in essentially all Grand Unified theories and/or superstring theories.

The "unification" predictions for gauge couplings rest on two premises. The first is that the $S U(3), S U(2)$ and $U(1)$ gauge couplings are related at the (large) unification scale and the second is that below this scale there is a specific multiplet content. The first premise follows if there is a stage of Grand Unification $\left(S U(5), S O(10), E_{6}\right.$ etc). However Grand Unification is not essential. In a (compactified) string theory the gauge couplings are related even if the gauge group is not unified. In this case the relationship between the couplings depends on the Kac Moody level used to construct the theory. In the simplest level-1 theory, the original string constructions yielded the "standard" $\mathrm{SU}(5)$ relationship between the gauge couplings even though the standard gauge group is just that of Standard Mode叫. Furthermore, in the case of the string theory the scale of gauge coupling unification is determined in terms of the only parameter of the theory, the string tension. Fixing this to obtain the correct gravitational strength coupling, one finds that the unification scale should be close to the Planck scale, i.e of $O\left(10^{18} \mathrm{GeV}\right)$. The fact that the unification of the gauge couplings of the Standard Model requires a very large scale of unification of $O\left((1-3) \times 10^{16}\right) \mathrm{GeV}$ may be considered to be the first indication that we are on the right track when seeking to extend unification to include gravity as in the string theory. However, one should note that the prediction of the scale of unification of the Standard Model gauge group couplings applies only to the class of string theory which do not have a stage of Grand Unification.

The second premise needed for the unification predictions is the choice of the low-energy spectrum below the unification scale. The usual "minimal" assumption is that this is just the minimal supermultiplet spectrum of the minimal supersymmetric Standard Model (MSSM). However this is not the most reasonable expectation.

In any viable Grand Unified theory it is necessary to add additional supermultiplets to obtain the required pattern of symmetry breaking. These additional multiplets are vectorlike with respect to the Standard Model group, so that they may obtain very large masses of the order of the (Grand) unification scale. However, only if they are exactly degenerate with the additional massive gauge bosons of the Grand Unified theory, will they leave the "minimal" unification predictions unchanged.

In string unification, even without Grand Unification, there is a definite multiplet content. We noted above that even if the gauge group is just that of that Standard Model, provided it is a level-1 string theory, the gauge couplings may still be related at the unification scale as in the prototype $S U(5)$ GUT. In this case, the breaking of the symmetry is through Wilson lines and one may show

\footnotetext{
${ }^{1}$ Subsequently, alternative constructions were developped which can lead to non-standard U(1) normalisation even in level-1 theory - for example see discussion in ref. [1]
} 
that the families of quarks and leptons belong to complete $S U(5)$ multiplets even though there is no residual $S U(5)$ gauge symmetry [2]; this is just as is required by observation. In addition, specific Wilson line breaking allows for an additional light pair of Higgs doublets as required in the MSSM. However, like the conventional GUT, such string theories usually have additional states, vectorlike under the Standard Model group, which can acquire masses of the order of the unification scale. In cases with a single Abelian Wilson line associated with a freely acting discrete symmetry, these states must fill out complete $S U(5)$ representations or come as additional copies of the Higgs sector; no other representations are allowed even though there is no Grand Unified group. It is worth remarking that the presence of complete degenerate $S U(5)$ multiplets does not affect the unification of gauge couplings at one loop and thus, the success of gauge unification applies in this case too. However, additional massive Higgs doublets do spoil the unification predictions at one loop and are disfavoured.

With the minimal spectrum of the MSSM and assuming the $S U(5)$ normalization of the $U(1)$ gauge couplings, one may determine the three gauge couplings in terms of the value of the unified coupling and the unification scale giving one overall prediction (up to uncertainties due to our lack of knowledge of the low energy SUSY spectrum). It is most convenient to express this as a prediction for the strong coupling, as this is the least well known of the three couplings. This gives $\alpha_{3}\left(M_{z}\right) \geq 0.126$ [3] including the supersymmetric threshold corrections [4], using as an input the well measured value of $\sin ^{2} \theta_{W}$ and $\alpha_{E M}$. This is in disagreement with the world average value of $\alpha_{3}\left(M_{z}\right)=0.118 \pm 0.003$ [5], albeit marginally so. In string theories, as we have already mentioned, the gauge unification scale is also predicted. In the case of the (weakly coupled) heterotic string the prediction is [6]

$$
M_{\text {string }} \approx g_{\text {string }} \times\left(5.2 \times 10^{17} \mathrm{GeV}\right) \approx 3.6 \times 10^{17} \mathrm{GeV}
$$

more than an order of magnitude larger than that predicted by the MSSM. Ways to resolve this discrepancy have been recently reviewed by Dienes [7]. Of particular interest is a recent suggestion of Witten [8] that the (10 dimensional) strongly coupled heterotic string theory ( $\mathrm{M}$ theory) gives a gauge coupling more closely in agreement with the gauge unification value.

It is clear that the precision measurements of the gauge couplings already allow for a very detailed test of the unification predictions and offer the potential of distinguishing between different unified theories. Given this, we consider it important to explore in detail the implications for the unification predictions of a non-minimal spectrum at the unification scale of the type to be expected in realistic extensions of the Standard Model. As we have mentioned, there are likely to be additional vectorlike states with masses of order of the unification scale belonging to complete $S U(5)$ multiplets or copies of the MSSM Higgs supermultiplets. Only if these states have exactly the unification scale mass, will the usual MSSM predictions apply, but the difference in the predictions if this is not the case applies in two loop order and may be expected to be small. We will consider in detail the effects of $(\overline{5}+5)$ and $(\overline{10}+10)$ additional $S U(5)$ supermultiplets which have masses less than the unification scale. While this does not exhaust the possibilities in Grand Unified theories, as there may be further higher dimension representations, the method we develop to obtain precision solutions to the renormalization group equations can readily be generalized to such representations. However, in the class of string theories discussed above, the only states allowed are indeed just those that fill out the five and ten dimensional representations, so the analysis presented here is quite general f $\mathrm{f}$. Furthermore, it is only in the context of such string theories that the prediction for the string unification scale may be directly compared with the unification scale of the couplings of the Standard Model.

The organization of the paper is as follows. In the next Section we discuss the mass splitting of the additional massive states. This is important because it gives rise to threshold corrections to the gauge

\footnotetext{
${ }^{2}$ We discard the possibility of additional massive Higgs representations, for they are known to spoil the unification predictions at one loop order.
} 
couplings. In the case of Grand Unification, the splitting comes from calculable radiative corrections and the resulting threshold corrections are of the same order as the usual two loop corrections. In the case of string unification without Grand Unification, the components of an $S U(5)$ multiplet are not related by the low energy symmetry and there may be additional threshold effects.

In Section 3 we develop a method for determining the low energy coupling $\alpha_{3}\left(M_{z}\right)$ and the unification scale, correct to two loop order in the running coupling, ignoring the effect of Yukawa couplings. The simplest method is just to integrate numerically the coupled differential equations, technique used in [9] and in [10]. However, we found problems in achieving the required accuracy using this technique, so we developed a method in which the calculation is done analytically. This has the advantage of more clearly exhibiting the important parameters in the calculation. In Section 4 we compare our intermediate results with the form presented by Shifman [11], derived by a completely different method, and show that they lead to same answer. In Section 5 we use the analysis to determine analytic formulae for the unification scale, the strong coupling constant and the intermediate scale masses. Using these formulae we determine the numerical predictions and present them in Section 6 . The effect of additional corrections to the mass of the heavy $S U(5)$ multiplets is considered in Section 7. Finally in Section 8 we present a summary of our results and our conclusions.

\section{The Massive spectrum}

We are interested in the class of models with additional vectorlike states $I+\bar{I}$ where $I, \bar{I}$ denote complete representations of $S U(5)$. The components of complete $S U(5)$ representations transform under $\mathrm{SU}(3)$ and $\mathrm{SU}(2)$ groups as follows: for $\psi=d^{c}:(\overline{3}, 1), \psi=l:(1,2)$ for the case $I$ is the 5 dimensional representation of $S U(5)$ and $\psi=e^{c}:(1,1), \psi=u^{c}:(\overline{3}, 1), \psi=q:(3,2)$ for the case $I$ is the 10 dimensional representation of $S U(5)$. We note that in string compactifications there are just such additional states left massless after compactification [2]. Here we wish to discuss how they obtain a (large) mass. Since they come in vectorlike combinations, we can write down $S U(3) \otimes S U(2) \otimes U(1)$ invariant mass terms $\mu_{\psi} \psi \bar{\psi}$ where the mass $\mu_{\psi}$ can be far above the electroweak breaking scale. This term will arise from a stage of spontaneous symmetry breaking through the coupling $\lambda_{\psi} \phi_{\psi} \psi \bar{\psi}$, when the $S U(3) \otimes S U\left(2 \otimes U(1)\right.$ invariant scalar field $\phi_{\psi}$ acquires a vacuum expectation value (vev). There is a very natural explanation for the origin of this vev, because the mass squared of the fields $\phi_{\psi}$ will be driven negative by the radiative corrections involving the coupling $\lambda_{\psi}$, in the usual radiative breaking mechanism [12].

To discuss this possibility in more detail, we consider first the case of a single $\phi$ with Yukawa coupling $\lambda_{\psi} \phi \psi \bar{\psi}$. The renormalization group equations have the form

$$
\begin{gathered}
\frac{d \alpha_{i}}{d t}=b_{i}^{\prime} \alpha_{i}^{2} \\
\frac{d \ln Y_{\psi}}{d t}=-2 \sum_{j=1}^{3} \eta_{j, \psi} \alpha_{j}+2 Y_{\psi}+\frac{n_{5}}{2}\left(3 Y_{d^{c}}+2 Y_{l}\right)+\frac{n_{10}}{2}\left(6 Y_{q}+3 Y_{u^{c}}+Y_{e^{c}}\right) \\
\frac{d m_{\phi}^{2}}{d t}=\sum_{\psi} s_{\psi} Y_{\psi}\left(m_{\phi}^{2}+m_{\psi}^{2}+\bar{m}_{\psi}^{2}\right) \\
\frac{d m_{\psi}^{2}}{d t}=-2 \sum_{j=1}^{3} \eta_{j, \psi} M_{j}^{2} \alpha_{j}+Y_{\psi}\left(m_{\phi}^{2}+m_{\psi}^{2}+\bar{m}_{\psi}^{2}\right)
\end{gathered}
$$

Here $\alpha_{i}=g_{i}^{2} / 4 \pi, i=\{1,2,3\}, Y_{\psi}=\lambda_{\psi}^{2} /(4 \pi), b_{i}^{\prime}=b_{i}+\left(n_{5}+3 n_{10}\right) / 2$ where $b_{i}$ is the MSSM one loop beta function, $\eta_{j, \psi}$ are given in the Appendix and $M_{i}$ are the gaugino masses which evolve 
as $M_{i}(t)=M_{i}(0)\left(\alpha_{i}(t) / \alpha_{i}(0)\right)$. Also $m_{\phi}, m_{\psi}$ and $\bar{m}_{\psi}$ refer to the soft supersymmetry breaking masses of the fields $\phi, \psi$ and $\bar{\psi}$ respectively ${ }^{\beta}$. The above sum over $\psi$ runs over the component fields $\psi=\left\{l, d^{c}, e^{c}, u^{c}, q\right\}$ for the case one considers both 5 and 10 dimensional complete representations of $S U(5)$ with $s_{\psi}=\frac{1}{2}\left\{2 n_{5}, 3 n_{5}, n_{10}, 3 n_{10}, 6 n_{10}\right\}$.

Let us discuss how the field $\phi$ actually acquires a vev, and what the expectation for its magnitude is. This requires us to solve the renormalization group equations for the soft SUSY breaking masses $m_{\phi}$, $m_{\psi}, \bar{m}_{\psi}$. It is straightforward to solve these equations numerically in specific cases. To determine the general feature, we solve a more simple example, keeping only the QCD gauge coupling and considering the case of a single component field $\psi$ which has QCD quantum numbers. Solving eqs (田),(司) leads to the result $m_{\psi}=\bar{m}_{\psi}$ and

$$
\begin{gathered}
m_{\phi}^{2}(t)=\left[\frac{\alpha(t)}{\alpha(0)}\right]^{\gamma_{m}}\left\{m_{\phi}^{2}(0)+\frac{2 s_{\psi} Y_{\psi}^{*}}{b_{3}^{\prime}\left(\gamma_{\psi}-\gamma_{m}\right)}\left[m_{\psi}^{2}(0)+\frac{2 \eta_{j, \psi} M_{3}^{2}(0)}{b_{3}^{\prime}\left(2-\gamma_{\psi}\right)}\right]\left[\left(\frac{\alpha(t)}{\alpha(0)}\right)^{\gamma_{\psi}-\gamma_{m}}-1\right]\right. \\
\left.-\frac{4 s_{\psi} Y_{\psi}^{*} \eta_{j, \psi} M_{3}^{2}(0)}{b_{3}^{\prime 2}\left(2-\gamma_{m}\right)\left(2-\gamma_{\psi}\right)}\left[\left(\frac{\alpha(t)}{\alpha(0)}\right)^{2-\gamma_{m}}-1\right]\right\}
\end{gathered}
$$

where $t=\frac{1}{2 \pi} \ln \left(\mu / M_{g}\right)$ and $\gamma_{m}=s_{\psi} Y_{\psi}^{*} / b_{3}^{\prime}, \gamma_{\psi}=2 Y_{\psi}^{*} / b_{3}^{\prime}$ where $Y_{\psi}^{*}$ denotes the fixed point value for the ratio $Y_{\psi} / \alpha_{3}$ which can be easily computed from eqs.(2) and (3). Note that this equation implies that $m_{\phi}^{2}$ will be driven negative by the radiative corrections, triggering a vacuum expectation value (vev) for $\phi$. This is the general behavior for our simplified example with one field $\psi$ only.

In the case that $\phi$ has a "flat" potential th, the scale $\Lambda$ at which this happens, corresponds closely to the vev that is induced for $\phi$. This then gives a mass to the vectorlike fields $I, \bar{I}$ coupled to $\phi$. The important point to note is that $\Lambda$ is determined by the multiplet content and by the allowed couplings of the theory; it is not a free parameter. In the case we are considering, it is easy to determine the order of magnitude of $\Lambda$ using that $\gamma_{\psi} \propto 1 / n_{10}\left(\right.$ or $\left.1 / n_{5}\right)$ and $\gamma_{m} \sim 1$. Hence, for large $n_{10}\left(n_{5}\right)$ case, appropriate to our use of the fixed points in solving these equations, and with $M_{3}(0) / m_{\psi}(0)<<1$, we get from eq. (6) that:

$$
m_{\phi}^{2}(t)=\left[\frac{\alpha(t)}{\alpha(0)}\right]^{\gamma_{m}}\left\{m_{\phi}^{2}(0)-2 m_{\psi}^{2}(0)\left[\left(\frac{\alpha(t)}{\alpha(0)}\right)^{-\gamma_{m}}-1\right]\right\}
$$

The point at which $m_{\phi}^{2}$ is driven negative is clearly dependent on the initial value of $m_{\phi} / m_{\psi}$. With this of order 1 , it will occur at the point where $\alpha(t) / \alpha(0)$ deviates from 1 by $O(1)$. For the case of large number of extra multiplets, this will be very close to the unification scale $M_{g}$, so $\Lambda$ will be close to this scale too.

Although, as we have just shown, it is possible that the intermediate scale breaking is triggered close to the unification scale, for $\alpha(t) / \alpha(0)$ of $O(1)$, it is also possible for a much smaller intermediate scale to be generated, corresponding to a much smaller ratio of $\alpha(t) / \alpha(0)$, its magnitude depending sensitively on this ratio.

Even if these parameters are of the same order, the intermediate scale may be much smaller than our initial estimate. If $\phi$ does not have a "flat" potential, then the scale of the vev of $\phi$ is not determined by the scale at which its mass squared becomes negative. To see this, let us assume the potential with leading term $\phi^{r} / M^{r-4}$, where $M$ is the scale of the new physics leading to the effective potential, which we take to be the compactification or Planck scale. Then, the vev of $\phi$ is proportional

\footnotetext{
${ }^{3}$ We use the notation $n_{5}=N_{5}+N_{\overline{5}}, n_{10}=N_{10}+N_{\overline{1} 0}$

${ }^{4} i$.e. there are no terms in the potential involving $\phi$.

${ }^{5}$ The value of $r$ will be determined by the symmetries of the theory.
} 
to $\left(m_{\phi}^{2} M^{r-4}\right)^{1 /(r-2)}$. Since the SUSY breaking scale $m_{\phi}$ is of $O(1 T e V)$, this implies an intermediate scale ranging from $10^{10} \mathrm{GeV}$ to $10^{15} \mathrm{GeV}$ for $r$ between 6 and 12 .

To summarize, there is a very natural origin for the generation of the intermediate scale, coming from radiative breaking. The magnitude of the intermediate scale is sensitively dependent on the details of the theory.

Let us now consider the expectation for individual intermediate scale masses.

For the case of Grand Unification, $S U(5)$ or larger, the mass terms must be $S U(5)$ invariant, so there is only a single field $\phi$ and $\lambda_{\psi}$ is independent of $\psi$. Thus, at the unification scale, all components $\psi$ of a representation $I$ are degenerate. However, below this scale, until the scale $M_{I}=\langle\phi\rangle$, radiative corrections will split the mass of the components. For the case when one can ignore the Yukawa couplings such as $\lambda_{\psi}$ in computing the radiative corrections, this splitting is generated by the gauge interactions only and is thus easily calculable. The effects of this splitting is included in the determination of the gauge unification predictions given in the next Section. The case where Yukawa couplings are not negligible is discussed below and in Section 7.

For the case of string unification, even without Grand Unification, the gauge couplings will be unified. Indeed, if the string prediction for the unification scale is relevant, there must not be a stage of Grand Unification because the prediction refers to the unification scale for the couplings of the gauge group at compactification scale, and this must just be that of the Standard Model. However, without Grand Unification, there may be different fields $\phi_{\psi}$ responsible for giving mass to the different components of the $S U(5)$ representation. Furthermore, there is no reason why the couplings $\lambda_{\psi}$ should be the same for all $\psi$ 's.

Let us first consider the case that there is only one field $\phi$. In this case the Yukawa coupling $\lambda_{\psi} \phi \psi \bar{\psi}$ leads to a mass $\lambda_{\psi}\langle\phi\rangle$ where $\langle\phi\rangle$ is the vev of $\phi$. The individual couplings $\lambda_{\psi}$ get renormalised according to eq(3) causing the spectrum to split. Just as for the $S U(5)$ case, the gauge contributions (the first term on the rhs) are included in our analysis below. The case the Yukawa couplings are large is discussed in Section 7. This leaves only the corrections which occur if the bare couplings $\lambda_{\psi}$ are different and the effect of this is also presented in Section 7.

Finally we consider the possibility when there are different fields $\phi_{\psi}$ responsible for the masses of the various components $\psi=\left\{l, d^{c}, e^{c}, u^{c}, q\right\}$ of the representations of $S U(5)$. Again radiative effects

will change the couplings $\lambda_{\psi}$. However, there is a more important effect, because the radiative breaking mechanism giving rise to the $\phi_{\psi}$ vev, drives $m_{\phi_{\psi}}$ negative at different scales, generating different vevs. The terms responsible for this in the renormalization group equations are those proportional to the gauge coupling strength of the component field $\psi$. The net effect of this is that the $(3,1)$ components of the 5 dimensional are heavier than the $(1,2)$ components, and the components in the 10 dimensional representation are similarly ordered with $(3,2)>(3,1)>(1,2)>(1,1)$. This effect is in the same direction as that driven by the gauge radiative corrections to the couplings alone, but generates a larger splitting between the components. Again the effect of this may be determined using the results of Section 7.

\section{Renormalization group equations}

We consider the two loop renormalization group equations for the gauge couplings, with no top, bottom and tau Yukawa interaction. Their general form is given by:

$$
\frac{d \alpha_{i}}{d t}=\tilde{b}_{i} \alpha_{i}^{2}+\frac{1}{4 \pi} \sum_{j=1}^{3} \tilde{b}_{i j} \alpha_{i}^{2} \alpha_{j}+\mathcal{O}\left(\alpha^{4}\right)
$$


with $i=\{1,2,3\}$ and where $t=\frac{1}{2 \pi} \log Q / M_{g} ; M_{g}$ is the unification scale, and $\tilde{b}_{i}$ and $\tilde{b}_{i j}$ are, in a general notation, the one loop and two loop beta functions respectively, which depend on the multiplet content of the theory. Dividing both sides by $\alpha_{i}^{2}$ we may rewrite this equation in the form

$$
d\left(-\alpha_{i}{ }^{-1}\right)=\tilde{b}_{i} d t+\frac{1}{4 \pi} \sum_{i=1}^{3} \frac{\tilde{b}_{i j}}{\tilde{b}_{j}} d\left(\ln \alpha_{j}\right)+\mathcal{O}\left(\alpha^{2}\right)
$$

where we have used $\alpha_{j} d t=\frac{1}{\tilde{b}_{j}} \frac{d \alpha_{j}}{\alpha_{j}}+\mathcal{O}\left(\alpha^{2}\right)$ to rewrite the second term on the right hand side. We integrate this equation from $M_{g}$ scale down to $M_{z}$ scale, and replace $\tilde{b}_{i}$ and $\tilde{b}_{i j}$ with their appropriate values. As discussed in the previous section, the mass splitting of the additional $S U(5)$ multiplets added to the MSSM spectrum are generated by radiative corrections. Thus to $\mathcal{O}\left(\alpha^{2}\right)$, the threshold effects coming from this splitting need only be included at one loop order; the two loop terms may be cutoff at the mean mass, $\mu_{q}$ of the multiplet. This gives

$$
\alpha_{i}^{-1}\left(M_{z}\right)=-\delta_{i}+\alpha_{g}^{-1}+\frac{b_{i}^{\prime}}{2 \pi} \ln \left[\frac{M_{g}}{M_{z}}\right]+\zeta_{i}+\frac{1}{4 \pi} \sum_{j=1}^{3} \frac{b_{i j}^{\prime}}{b_{j}^{\prime}} \ln \left[\frac{\alpha_{g}}{\alpha_{j}\left(\mu_{q}\right)}\right]+\frac{1}{4 \pi} \sum_{j=1}^{3} \frac{b_{i j}}{b_{j}} \ln \left[\frac{\alpha_{j}\left(\mu_{q}\right)}{\alpha_{j}\left(M_{z}\right)}\right]
$$

with

$$
\zeta_{i}=-\frac{1}{2 \pi} \sum_{\psi} N_{\psi} \beta_{i, \psi} \ln \left[\frac{\mu_{\psi}\left(\mu_{\psi}\right)}{M_{z}}\right]
$$

In the above equations $\delta_{i}$ stands for low energy supersymmetric thresholds; we will express our results as a change of the MSSM predictions following from the addition of heavy states and in this the $\delta_{i}$ cancel; further, $\psi$ stands for the component fields of additional complete $S U(5)$ multiplets we consider and runs over $\left\{l, d^{c}, e^{c}, u^{c}, q\right\}$; their mass is $\mu_{\psi} ; b_{i}^{\prime}$ and $b_{i j}^{\prime}$ are the one loop and two loop beta functions when none of these states is decoupled[f; their expressions are presented in the Appendix. The above formula is correct to two loop order.

As we have stressed, the one loop threshold effects $\zeta_{i}$ are in fact of the same order as the two loop terms. This is made explicit from the one loop renormalization group equations for the running mass

$$
\frac{d \mu_{\psi}}{d t}=-\mu_{\psi} \sum_{j=1}^{3} \alpha_{j} \eta_{j, \psi}
$$

where $\eta_{j, \psi}$ are given in the Appendix and we have ignored Yukawa interactions involving the $\psi$. Keeping terms to $\mathcal{O}\left(\alpha^{2}\right)$ corresponds to keeping only the one loop running approximation for the couplings, in the presence of extra-matter:

$$
\frac{d \alpha_{i}}{d t}=b_{i}^{\prime} \alpha_{i}^{2}+\mathcal{O}\left(\alpha^{3}\right)
$$

Using this we may integrate eq(12) to get

$$
\ln \left[\frac{\mu_{\psi}\left(\mu_{\psi}\right)}{\mu_{g}}\right]=\sum_{j=1}^{3} \frac{\eta_{j, \psi}}{b_{j}^{\prime}} \ln \left[\frac{\alpha_{g}}{\alpha_{j}\left(\mu_{\psi}\right)}\right]
$$

where we have assumed that all the heavy states get a common mass $\mu_{g}$ at unification scale. One sees explicitly that one loop threshold effects are, in fact, two loop-like effects. As before we may further

\footnotetext{
${ }^{6}$ We assume here that all additional states of a given flavour are degenerate; it is easy to generalise the method to the non-degenerate case if desired.
} 
simplify this equation to write

$$
\ln \left[\frac{\mu_{\psi}\left(\mu_{\psi}\right)}{\mu_{g}}\right]=\sum_{j=1}^{3} \frac{\eta_{j, \psi}}{b_{j}^{\prime}} \ln \left[\frac{\alpha_{g}}{\alpha_{j}\left(\mu_{q}\right)}\right]
$$

for any $\psi$, since the effect of the radiative mass difference between different flavours generates terms of higher order.

Using eq. (15) to express $\zeta_{i}$ given in eq.(11), we get from eq.(10) the value of the couplings at $M_{z}$ scale:

$$
\begin{aligned}
\alpha_{i}^{-1}\left(M_{z}\right)= & -\delta_{i}+\alpha_{g}^{-1}+\frac{b_{i}}{2 \pi} \ln \left[\frac{M_{g}}{M_{z}}\right]+\frac{n}{2 \pi} \ln \left[\frac{M_{g}}{\mu_{g}}\right]-\frac{1}{2 \pi} \sum_{j=1}^{3} Y_{i j} \ln \left[\frac{\alpha_{g}}{\alpha_{j}\left(\mu_{q}\right)}\right] \\
& +\frac{1}{4 \pi} \sum_{j=1}^{3} \frac{b_{i j}}{b_{j}} \ln \left[\frac{\alpha_{g}}{\alpha_{j}\left(M_{z}\right)}\right]
\end{aligned}
$$

where

$$
Y_{i j}=\frac{n}{b_{j}^{\prime}}\left[\frac{1}{2} \frac{b_{i j}}{b_{j}}-\delta_{i j} \lambda_{j}\right]
$$

and $\lambda_{1}=0, \lambda_{2}=2, \lambda_{3}=3$.

These equations demonstrate an interesting result. At one loop the running of the couplings depends only on the number of additional states through the combination $n=\left(n_{5}+3 n_{10}\right) / 2$. We see that is also true at two loop, even though it is not true for the two loop beta functions, because of the threshold effects. Note that this only applies in the case Yukawa couplings are ignored. Note also the mild dependence of the functions $Y_{i j}$ on $n$.

In order to evaluate the effect of the additional heavy states, it is convenient to compare the results with heavy states to the minimal MSSM predictions. In the latter, the Renormalization Group Equations (RGE) are

$$
\alpha_{i}^{o-1}\left(M_{z}\right)=-\delta_{i}^{o}+\alpha_{g}^{o-1}+\frac{b_{i}}{2 \pi} \ln \left[\frac{M_{g}^{o}}{M_{z}}\right]+\frac{1}{4 \pi} \sum_{j=1}^{3} \frac{b_{i j}}{b_{j}} \ln \left[\frac{\alpha_{g}^{o}}{\alpha_{j}^{o}\left(M_{z}\right)}\right]
$$

again ignoring Yukawa couplings. The MSSM variables are labelled with an "o" index to distinguish them from the model which considers additional complete $\mathrm{SU}(5)$ multiplets. We follow the general approach and fix, as an input, the values for $\alpha_{1}\left(M_{z}\right)$ and $\alpha_{2}\left(M_{z}\right)$ which are known with a good accuracy from the experimental values for $\alpha_{e m}\left(M_{z}\right)$ and $\sin \theta_{W}\left(M_{z}\right)$. This fixes the values for $\alpha_{g}^{o}$ and $M_{g}^{o}$ which are then used to compute numerically the value for $\alpha_{3}^{o}\left(M_{z}\right)$. As the MSSM has been extensively studied numerically, we can take all these MSSM quantities as known.

Note that the parameters $\delta_{i}^{o}$ which appear in eq(18) and the parameters $\delta_{i}$ which appear in eq(11) include the constant factors needed for $\overline{M S} \rightarrow \overline{D R}$ (which are the same in MSSM and our model घ), the low energy supersymmetry thresholds effects and contributions from nonrenormalizable operators at the high scale [13]. The SUSY threshold effects are most conveniently included in terms of the effective scale $T_{\text {susy }}^{o}$ 14 relevant when calculating the strong coupling in terms of the weak and electromagnetic couplings. Thus, when comparing the prediction with heavy states to the MSSM prediction we find

$$
\alpha_{3}^{-1}\left(M_{z}\right)=\alpha_{3}^{o-1}\left(M_{z}\right)+\Delta \alpha_{3}^{-1}\left(M_{z}\right)
$$

\footnotetext{
${ }^{7}$ and will therefore cancel in the calculation below
} 
where the SUSY threshold effects give a contribution

$$
\Delta \alpha_{3}^{-1}\left(M_{z}\right)=\frac{19}{28 \pi} \ln \left[\frac{T_{\text {susy }}}{T_{\text {susy }}^{o}}\right]
$$

and $T^{\text {susy }}$ is the new effective scale in MSSM plus the extra heavy states. At one-loop order, the SUSY threshold factors are independent of the gauge couplings and thus are the same for the MSSM and the MSSM with additional heavy states. At two loop order, the SUSY threshold factors do depend on gauge couplings, but the difference between these couplings in the models with heavy states is $O(\alpha)$ and thus the net effect is beyond the two-loop order and can be neglected. The same arguments enable us to avoid the explicit form of SUSY thresholds when computing other variables of the model with heavy multiplets, like $M_{g}$ or $\mu_{\psi}\left(\mu_{\psi}\right)$.

\section{Compatibility with other approaches.}

After completing this analysis our attention was brought to the analysis of Shifman [11] derived from a different point of view using holomorphicity arguments and the instanton calculus. Here we wish to compare the results of the two approaches, namely the comparison of eq.(16) with Shifman's "master formula" [11]. We reproduce it below (ignoring the heavy Higgs triplet and 24-plet contributions [5, but including the other heavy states' contributions; we also add some generic values, $\delta_{i}$, for the low energy Susy thresholds, as we want the values of couplings at $M_{z}$ scale. We have:

$$
\begin{aligned}
\alpha_{1}^{-1}\left(M_{z}\right)= & -\delta_{1}+\alpha_{g}^{-1}+\frac{1}{2 \pi} \frac{3}{10}\left\{\sum _ { g e n } \left[\ln \frac{M_{g}}{M_{z} Z_{l L}}+2 \ln \frac{M_{g}}{M_{z} Z_{e R}}+\frac{1}{3} \ln \frac{M_{g}}{M_{z} Z_{q L}}\right.\right. \\
& \left.\left.+\frac{8}{3} \ln \frac{M_{g}}{M_{z} Z_{u R}}+\frac{2}{3} \ln \frac{M_{g}}{M_{z} Z_{d R}}\right]+2 \ln \frac{M_{g}}{M_{z} Z_{H_{u, d}}}\right\}+\frac{n}{2 \pi} \ln \frac{M_{g}}{\mu_{g}} \\
\alpha_{2}^{-1}\left(M_{z}\right)= & -\delta_{2}+\alpha_{g}^{-1}-\frac{6}{2 \pi} \ln \frac{M_{g}}{M_{z}\left(\frac{\alpha_{g}}{\alpha_{2}\left(M_{z}\right)}\right)^{1 / 3}}+\frac{1}{2 \pi} \sum_{g e n}\left[\frac{3}{2} \ln \frac{M_{g}}{M_{z} Z_{q L}}+\frac{1}{2} \ln \frac{M_{g}}{M_{z} Z_{l L}}\right] \\
+ & \frac{1}{2 \pi} \ln \frac{M_{g}}{M_{z} Z_{H_{u, d}}}+\frac{n}{2 \pi} \ln \frac{M_{g}}{\mu_{g}} \\
\alpha_{3}^{-1}\left(M_{z}\right)= & -\delta_{3}+\alpha_{g}^{-1}-\frac{9}{2 \pi} \ln \frac{M_{g}}{M_{z}\left(\frac{\alpha_{g}}{\alpha_{3}\left(M_{z}\right)}\right)^{1 / 3}}+\frac{1}{2 \pi} \sum_{g e n}\left[\ln \frac{M_{g}}{M_{z} Z_{q L}}+\frac{1}{2} \ln \frac{M_{g}}{M_{z} Z_{u R}}\right. \\
& \left.+\frac{1}{2} \ln \frac{M_{g}}{M_{z} Z_{d R}}\right]+\frac{n}{2 \pi} \ln \frac{M_{g}}{\mu_{g}}
\end{aligned}
$$

The advantage of this form for the running of gauge couplings is that (above supersymmetric scale, when $\delta_{i}=0$ ) this form is exact to all orders. However the wave function renormalization coefficients $Z$ are only known perturbatively so one is still confined to a given order in perturbation theory when testing coupling unification. To two loop order, one must include the values of wave-function renormalization coefficients $Z$ in one loop only; also, replacing them with their two loop value would

\footnotetext{
${ }^{8}$ These are not included in this work.
} 
give a three loop calculation and so on 9 . Here we only show that, using their one loop values, we get the same two loop form for the running of gauge couplings as we got in equation (16). The $Z$ coefficients are, to one loop, given by:

$$
Z_{S U(j)}=\left[\frac{\alpha_{g}}{\alpha_{j}\left(\mu_{q}\right)}\right]^{-\frac{2 C_{2}}{b_{j}^{\prime}}}\left[\frac{\alpha_{j}\left(\mu_{q}\right)}{\alpha_{j}\left(M_{Z}\right)}\right]^{-\frac{2 C_{2}}{b_{j}}}=\left[\frac{\alpha_{g}}{\alpha_{j}\left(\mu_{q}\right)}\right]^{\frac{2 C_{2}}{b_{j}^{\prime}} \frac{n}{b_{j}}}\left[\frac{\alpha_{g}}{\alpha_{j}\left(M_{Z}\right)}\right]^{-\frac{2 C_{2}}{b_{j}}}
$$

where $j=1,2,3, C_{2}$ is the second order Casimir operator 0 for $S U(N)$, and $b_{j}^{\prime}=b_{j}+n$ and where we took into consideration the one loop renormalization of these coefficients. Note that we can neglect the splitting of the heavy $S U(5)$ multiplets in the renormalization of the $Z$ factors as they correspond

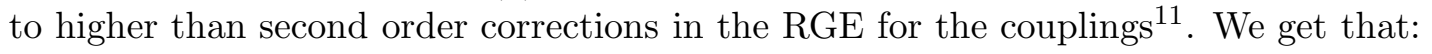

$$
\begin{aligned}
& Z_{S U(3)}=\left[\frac{\alpha_{g}}{\alpha_{3}\left(\mu_{q}\right)}\right]^{-\frac{8}{9} \frac{n}{b_{3}^{\prime}}}\left[\frac{\alpha_{g}}{\alpha_{3}\left(M_{Z}\right)}\right]^{\frac{8}{9}} \\
& Z_{S U(2)}=\left[\frac{\alpha_{g}}{\alpha_{2}\left(\mu_{q}\right)}\right]^{\frac{3}{2} \frac{n}{b_{2}^{\prime}}}\left[\frac{\alpha_{g}}{\alpha_{2}\left(M_{Z}\right)}\right]^{-\frac{3}{2}} \\
& Z_{U(1)}=\left[\frac{\alpha_{g}}{\alpha_{1}\left(\mu_{q}\right)}\right]^{\frac{2}{11} \frac{n}{b_{1}^{\prime}}}\left[\frac{\alpha_{g}}{\alpha_{1}\left(M_{Z}\right)}\right]^{-\frac{2}{11}}
\end{aligned}
$$

Also we have that

$$
\begin{gathered}
Z_{q L}=Z_{S U(3)} \times Z_{S U(2)} \times Z_{U(1)}^{1 / 36} \\
Z_{u R}=Z_{S U(3)} \times Z_{U(1)}^{4 / 9} \\
Z_{d R}=Z_{S U(3)} \times Z_{U(1)}^{1 / 9} \\
Z_{l L}=Z_{S U(2)} \times Z_{U(1)}^{1 / 4} \\
Z_{H_{u, d}}=Z_{S U(2)} \times Z_{U(1)}^{1 / 4} \\
Z_{e R}=Z_{U(1)}
\end{gathered}
$$

With these expressions for $Z$ coefficients we get from the "master formula" that:

$$
\begin{aligned}
\alpha_{i}^{-1}\left(M_{z}\right)= & -\delta_{i}+\alpha_{g}^{-1}+\frac{b_{i}}{2 \pi} \ln \left[\frac{M_{g}}{M_{z}}\right]+\frac{n}{2 \pi} \ln \left[\frac{M_{g}}{\mu_{g}}\right]+\frac{1}{4 \pi} \sum_{j=1}^{3} \frac{n}{b_{j}^{\prime}}\left[2 \delta_{i j} \lambda_{j}-\frac{b_{i j}}{b_{j}}\right] \ln \left[\frac{\alpha_{g}}{\alpha_{j}\left(\mu_{q}\right)}\right] \\
& +\frac{1}{4 \pi} \sum_{j=1}^{3} \frac{b_{i j}}{b_{j}} \ln \left[\frac{\alpha_{g}}{\alpha_{j}\left(M_{z}\right)}\right]
\end{aligned}
$$

where the $b_{i j}, b_{j}$ above are just the values of two loop and one loop beta functions for MSSM. This is exactly the formula we previously obtained in eq.(16).

\footnotetext{
${ }^{9}$ Beyond two loop order this form is regularisation scheme dependent 15 .

${ }^{10}$ divided by $Y^{2}$ for $\mathrm{U}(1)$, hence giving a $3 / 5$ factor, with $Y$ defined as in the Appendix; our definition for $Y$ differs from that of [11] and hence the different powers for $Z_{U(1)}$ in the following equations

${ }^{11}$ Strictly speaking one should use the bare mass $\mu_{g}$ in the expressions for $Z$ instead of $\mu_{q}$, but again, this difference is of higher order.
} 


\section{Analytical Results}

We now proceed to solve the two loop RGE eqs.(16) analytically to obtain predictions for the model with additional $S U(5)$ multiplets. In both the analysis of the minimal MSSM unification and the non-minimal case we adopt, we follow the usual analysis method of using the measured weak and electromagnetic couplings to determine the strong coupling and the unification scale. Thus, we fix as input parameters at the full two loop order $\alpha_{1}\left(M_{z}\right)=\alpha_{1}^{o}\left(M_{z}\right)$ and $\alpha_{2}\left(M_{z}\right)=\alpha_{2}^{o}\left(M_{z}\right)$ and equal to their experimental value [12. In the case of $n$ additional $S U(5)$ heavy multiplets, the mean mass of these multiplets introduces a further parameter which we choose to be $\alpha_{g}$. The output parameters of our model will be the unification scale, $M_{g}$, the value of alpha strong at the $M_{z}$ scale, $\alpha_{3}\left(M_{z}\right)$, the masses of the additional heavy $S U(5)$ multiplets $\mu_{\psi}$ as well as their common mass $\mu_{g}$, at the unification scale. Thus, making use of eq.(18), we solve analytically, to two loop order [3], the system of equations (15), (16), (17) for the unknowns $M_{g}, \mu_{g}, \mu_{\psi}$, and $\alpha_{3}(M z)$ in terms of MSSM variables $M_{g}^{o}, \alpha_{3}^{o}\left(M_{z}\right)$ and $\alpha_{g}^{o}$. This will avoid any numerical evaluation of the system of these equations, as well as the inconveniences deriving from possible numerical instabilities.

To achieve this, we subtract eq.(18) from eq.(16) for same $i$ and obtain the following relation:

$$
\begin{aligned}
\alpha_{i}^{-1}\left(M_{z}\right)-\alpha_{i}^{o-1}\left(M_{z}\right)= & \alpha_{g}^{-1}-\alpha_{g}^{o-1}+\frac{b_{i}}{2 \pi} \ln \left[\frac{M_{g}}{M_{g}^{o}}\right]+\frac{n}{2 \pi} \ln \left[\frac{M_{g}}{\mu_{g}}\right]-\frac{1}{2 \pi} \sum_{j=1}^{3} Y_{i j} \ln \left[\frac{\alpha_{g}}{\alpha_{j}\left(\mu_{q}\right)}\right] \\
& +\frac{1}{4 \pi} \sum_{j=1}^{3} \frac{b_{i j}}{b_{j}} \ln \left[\frac{\alpha_{g}}{\alpha_{g}^{o}}\right]-\frac{1}{4 \pi} \frac{b_{i 3}}{b_{3}} \ln \left[\frac{\alpha_{3}\left(M_{z}\right)}{\alpha_{3}^{o}\left(M_{z}\right)}\right]
\end{aligned}
$$

Consider now the equations obtained from the above equation for $i=1$ and $i=2$, and subtract them to get:

$$
\begin{gathered}
0=\frac{b_{1}-b_{2}}{2 \pi} \ln \left[\frac{M_{g}}{M_{g}^{o}}\right]-\frac{1}{2 \pi} \sum_{j=1}^{3}\left(Y_{1 j}-Y_{2 j}\right) \ln \left[\frac{\alpha_{g}}{\alpha_{j}\left(\mu_{q}\right)}\right]+\frac{1}{4 \pi} \sum_{j=1}^{3} \frac{b_{1 j}-b_{2 j}}{b_{j}} \ln \left[\frac{\alpha_{g}}{\alpha_{g}^{o}}\right] \\
-\frac{1}{4 \pi} \frac{b_{13}-b_{23}}{b_{3}} \ln \left[\frac{\alpha_{3}\left(M_{z}\right)}{\alpha_{3}^{o}\left(M_{z}\right)}\right]
\end{gathered}
$$

The eq. (29) will give us the ratio $\frac{M_{g}}{M_{g}^{o}}$ (see later). The eq. (28) for $i=1$ will give us the value of the variable $\xi=1 /(2 \pi) \ln \left(M_{g} / \mu_{q}\right)$ which we introduce instead of $\mu_{q}$ as an independent variable and which proves useful in solving the system. To see this we make use of the eq.(15) considered for $\psi=q$ :

$$
-2 \pi \xi-\ln \left[\frac{\mu_{g}}{M_{g}}\right]=\sum_{j=1}^{3} \frac{\eta_{j, \psi}}{b_{j}^{\prime}} \ln \left[\frac{\alpha_{g}}{\alpha_{j}\left(\mu_{q}\right)}\right]
$$

to substitute the ratio $\frac{\mu_{g}}{M_{g}}$ in eq.(28). With the above three equations we compute $M_{g} / M_{g}^{o}, \mu_{g} / M_{g}$ (and therefore $\mu_{g} / M_{g}^{o}$ ) and $\xi$ in function of the remaining terms present in the above equations. We also observe that $\ln \left(\alpha_{3}\left(M_{z}\right) / \alpha_{3}^{o}\left(M_{z}\right)\right)=0$ in two loop order - hence we can drop it - as we can see it from the fact that $\alpha_{g}^{-1}-\alpha_{g}^{o-1}+n \xi=0$ in one loop order. We also extensively use that $\ln \left(\alpha_{g} / \alpha_{j}\left(\mu_{q}\right)\right)=\ln \left(1+\alpha_{g} b_{j}^{\prime} \xi\right)$ which is correct to two loop order. We therefore end up with expressions for $M_{g} / M_{g}^{o}, \mu_{g} / M_{g}^{o}$ in terms of $\xi$ and with a nonlinear equation for $\xi$ itself. On this latter equation

\footnotetext{
${ }^{12}$ The couplings are normalized such as $\alpha_{1}=\alpha_{2}=\alpha_{3}$ at the unification scale.

${ }^{13}$ This means that, in the following, we can discard terms which originate from three loop order in either MSSM or in the model with additional multiplets.
} 
we again observe that $\alpha_{g}^{-1}-\alpha_{g}^{o-1}+n \xi=0$ in one loop order as all log's present are two-loop or higher order log's. Hence the $\xi$ dependence of $M_{g} / M_{g}^{o}, \mu_{g} / M_{g}^{o}$ is finally lost, and the three equations decouple, as we can always replace $\ln \left(1+\alpha_{g} b_{j}^{\prime} \xi\right)$ by its corresponding value obtained for a $\xi$ computed in one loop. 四

To solve for $\alpha_{3}\left(M_{z}\right)$ we simply multiply eq.(28) for $i=1,2,3$ by $b_{2}-b_{3}, b_{3}-b_{1}$ and $b_{1}-b_{2}$ respectively, add them together and make use of the above observations. In all these processes the difference $\delta_{i}-\delta_{i}^{o}$ is dropped as it would bring a higher order contribution, as we mentioned in the previous section.

Finally, the remaining masses of the fields $\psi$ can be computed easily from equation (15).

After this algebra and consistent neglect of higher order terms, which proves to be essential in simplifying the equations, we get the following analytical results:

$$
\begin{gathered}
\frac{M_{g}}{M_{g}^{o}}=\left[\frac{\alpha_{g}}{\alpha_{g}^{o}}\right]^{\frac{31}{21}} \prod_{j=1}^{3}\left\{1+\frac{b_{j}^{\prime}}{n}\left[\frac{\alpha_{g}}{\alpha_{g}^{o}}-1-\frac{1168}{231 \pi} \alpha_{g} \ln \left[\frac{\alpha_{g}}{\alpha_{g}^{o}}\right]\right]\right\}^{\rho_{j}} \\
\alpha_{3}^{-1}\left(M_{z}\right)=\alpha_{3}^{o-1}\left(M_{z}\right)-\frac{470}{77 \pi} \ln \left[\frac{\alpha_{g}}{\alpha_{g}^{o}}\right]+\sum_{j=1}^{3} \ln \left\{1+\frac{b_{j}^{\prime}}{n}\left[\frac{\alpha_{g}}{\alpha_{g}^{o}}-1-\frac{1168}{231 \pi} \alpha_{g} \ln \left[\frac{\alpha_{g}}{\alpha_{g}^{o}}\right]\right]\right\}^{\omega_{j}} \\
\frac{\mu_{g}}{M_{g}^{o}}=\left[\frac{\alpha_{g}}{\alpha_{g}^{o}}\right]^{\frac{31}{21}+\frac{2336}{231 n}} \exp \left[\frac{2 \pi}{n}\left(\alpha_{g}^{-1}-\alpha_{g}^{o-1}\right)\right] \prod_{j=1}^{3}\left\{1+\frac{b_{j}^{\prime}}{n}\left[\frac{\alpha_{g}}{\alpha_{g}^{o}}-1-\frac{1168}{231 \pi} \alpha_{g} \ln \left[\frac{\alpha_{g}}{\alpha_{g}^{o}}\right]\right]\right\}^{\sigma_{j}} \\
\frac{\mu_{\psi}}{M_{g}^{o}}=\frac{\mu_{g}}{M_{g}^{o}} \prod_{j=1}^{3}\left\{1+\frac{b_{j}^{\prime}}{n}\left[\frac{\alpha_{g}}{\alpha_{g}^{o}}-1-\frac{1168}{231 \pi} \alpha_{g} \ln \left[\frac{\alpha_{g}}{\alpha_{g}^{o}}\right]\right]\right\}^{\tau_{j, \psi}}
\end{gathered}
$$

The values of $\rho_{j}, \omega_{j}, \sigma_{j}, \tau_{j, \psi}$, with $j=\{1,2,3\}$ and $\psi=\left\{l, d^{c}, e^{c}, u^{c}, q\right\}$, are given by

$$
\begin{gathered}
\rho_{j}=\left\{\frac{1}{12} \frac{n}{b_{1}^{\prime}}, \frac{-39}{28} \frac{n}{b_{2}^{\prime}}, \frac{4}{21} \frac{n}{b_{3}^{\prime}}\right\} \\
\omega_{j}=\left\{\frac{-2}{11 \pi} \frac{n}{b_{1}^{\prime}}, \frac{81}{14 \pi} \frac{n}{b_{2}^{\prime}}, \frac{2}{7 \pi} \frac{n}{b_{3}^{\prime}}\right\} \\
\sigma_{j}=\left\{\frac{11 n-7}{132 b_{1}^{\prime}}, \frac{-3(111+13 n)}{28 b_{2}^{\prime}}, \frac{4(22+n)}{21 b_{3}^{\prime}}\right\}
\end{gathered}
$$

and finally,

$$
\tau_{j, \psi}=\frac{\eta_{j, \psi}}{b_{j}^{\prime}}
$$

The values of $\eta_{j, \psi}$ and $b_{j}^{\prime}$ appearing above are given in the Appendix.

This gives the full analytical solution, to two loop order, to the system of non-linear equations (15), (16), (17), which contains three RGE equations and five equations for the running of the masses of $\psi$ 's.

The term $1168 / 231 / \pi \ln \left(\alpha_{g} / \alpha_{g}^{o}\right)$ in the above expressions can be dropped, as it brings in a higher order (three loop) correction; however, we keep it, as it gives an idea of the size of three loop corrections and makes a better agreement with the numerical approach of this problem. It is also relevant

\footnotetext{
${ }^{14}$ We will retain, however, a two loop term in the final results, to get an idea of the importance of three loop contributions
} 
when considering unification at strong $\alpha_{g}$. This is equivalent to considering that $\alpha_{g}^{-1}-\alpha_{g}^{o-1}+n \xi=$ $-1168 / 231 / \pi \ln \left(\alpha_{g} / \alpha_{g}^{o}\right)$ where the right hand side of the latter is a two loop correction 15 .

The solution demonstrates explicitly that the result depends on the number of additional states only, via the parameter $n=\left(n_{5}+3 n_{10}\right) / 2$. Also, the values of $\alpha_{3}\left(M_{z}\right)$ as well as the ratio $\frac{M_{g}}{M_{g}^{o}}$ depend, to two loop, on the ratio of the couplings at unification scale only. As a check of our calculation, one can take the limit $\alpha_{g} / \alpha_{g}^{o} \rightarrow 1$ in the above expressions to recover the MSSM case.

\section{$6 \quad$ Numerical results}

In this section we consider the numerical results given by the expressions of eqs. (31), (32), (33), (34).

For $n=1,2$ the ratio $M_{g} / M_{g}^{o}$ is rather small, though larger than unity; in the meantime $\alpha_{3}\left(M_{z}\right)$ is always larger than its MSSM value; it cannot became smaller than $\alpha_{3}^{o}\left(M_{z}\right)$ because $\square$, in that case, the additional states would be too light $(\approx 10-100 \mathrm{GeV})$ and these cases are therefore disfavoured. Moreover in these cases the two loop approximation breaks down for $\alpha_{g}$ quite close to its MSSM value, because the two loop contributions involve log's of very small arguments, which make their contribution dominate over the one loop contribution.

The case $n=3$ is special because the step used in deriving eq(9) breaks down as it involves division by a one loop beta function which is actually 0 . This case should be considered separately, even though, interesting enough, the limit $n=3$ in our final results exists and is finite, predicting a larger unification scale, up to a factor of $\approx 10$ for unification at strong coupling together with a larger $\alpha_{3}\left(M_{Z}\right)$. However the higher order corrections are expected to be very large in this case too so these results should be viewed with caution. This case has been analysed in detail [16], but ignoring the gauge corrections to thresholds which, as we have seen, affects the result considerably.

For $n=4$ or larger the perturbation expansion is well behaved for intermediate values of the coupling. The results for the unification scale and the strong coupling are shown in Figs 1 and 2. One may see that the unification scale is increased, but by a factor $<5$ for $\alpha_{g}<0.45$. The values of $\alpha_{3}\left(M_{z}\right)$ always increases and are larger than in MSSM. This is in disagreement with the analysis of [10] due, we think, to errors in the numerical intergration used. In fact it can be shown analytically that the partial derivative of $\alpha_{3}$ with respect to the ratio $\alpha_{g} / \alpha_{g}^{o}$ is always positive for any fixed $n \geq 4$ with $\alpha_{g} \geq \alpha_{g}^{o}$ (see its expression given in the Appendix). Therefore, in these cases, the strong coupling is larger then in MSSM.

The dependence of the masses of the additional states on the unified coupling is shown in Figs. 3, 4 and 5. One may see the masses increase as we increase the number of states $n$, while keeping $\alpha_{g}$ fixed. Note also that for a given $n$, there are two values of $\alpha_{g}$ which predict the same value of $\mu_{q}$, which shows the advantage of setting $\alpha_{g}$ as our input parameter instead of $\mu_{g}$, avoiding the ambiguity of the methods having $\mu_{g}$ as an input parameter and $\alpha_{g}$ as an output. This is particularly relevant when we consider the predictions for large values of the unified coupling.

To summarize, in contrast to previous analyses, we have found that the addition of heavy states systematically increase $\alpha_{3}\left(M_{z}\right)$ taking it further from the current experimental value. The effect of the additional states does increase the unification scale, but by a factor less than $\approx 5$ even for the largest couplings which may still be in the perturbative domain. Since for unified couplings of $O(0.3)$ the string prediction of eq(11) increases by a factor 2.7 there is only a small improvement with the weakly coupled heterotic string prediction. Note that the analysis has neglected the Yukawa couplings of the third generation which may be important; their effect is investigated elsewhere [17].

\footnotetext{
${ }^{15}$ See Appendix for a full two loop equation

${ }^{16}$ We use $\alpha_{3}^{o}\left(M_{z}\right)=0.125$
} 


\section{$7 \quad$ Massive threshold corrections}

The analytic results we obtained in Sections 5, 6 refer to the case when the heavy multiplets have a common bare mass, $\mu_{g}$ at the unification scale and are split only by gauge interactions. We found that the threshold corrections introduced by this splitting largely cancel the two-loop corrections in the beta functions leading to the form of eq(16) or equivalently eq(22). However Yukawa couplings will also cause the massive spectrum to split introducing further threshold corrections. Fortunately the form derived by Shifman, eq(22) shows that the cancellation persists for Yukawa corrections as well and that the heavy states contribute with their bare mass only; radiative corrections to the masses should not be included if the appropriate two-loop beta function is used. Thus the only effects of Yukawa couplings are contained in the $Z$ factors for the light fields. This is important because, unlike the threshold effects, these corrections are not proportional to $n$ and so we do not expect such effects to be large. There remains the corrections which may occur in non-Grand-Unified models due to the possible splitting of the bare Yukawa couplings $\lambda_{\psi}$ introduced in Section 2. In this case the correction factors to our previous results are given by

$$
\frac{M_{g}}{M_{g}^{o}}=\left[\frac{M_{g}}{M_{g}^{o}}\right]_{o l d}\left[\frac{\mu_{d^{c}}^{o}}{\mu_{l}^{o}}\right]^{\frac{n_{5}}{28}}\left[\frac{\mu_{e^{c}}^{o 3} \mu_{u^{c}}^{o 4}}{\mu_{q}^{o 7}}\right]^{\frac{n_{10}}{28}}
$$

where the label "old" used in this section refers to our previously obtained results of Sections 5 and 6 and $\mu_{\psi}^{o}$ stands for the bare mass for $\psi$ field. Also:

$$
\alpha_{3}^{-1}\left(M_{z}\right)-\alpha_{3}^{-1}\left(M_{z}\right)_{o l d}=\frac{9 n_{5}}{28 \pi} \ln \left[\frac{\mu_{l}^{o}}{\mu_{d^{c}}^{o}}\right]+\frac{3 n_{10}}{28 \pi} \ln \left[\frac{\mu_{q}^{o 7}}{\mu_{u^{c}}^{o 5} \mu_{e^{c}}^{o 2}}\right]
$$

for the change in the strong coupling. The general effect of this uncertainty requires a specific model for the bare couplings. However a general feature emerges. For the case of additional $5 s$ an increase in the unification scale is always accompanied by an increase in the strong coupling. For the case of additional $10 \mathrm{~s}$ it is possible to avoid this, but only through a very special choice of the bare masses which, lacking a specefic model, looks quite unnatural. Further one may see that the change in the unification scale is quite small for reasonable numbers of additional multiplets unless the bare masses are split by many orders of magnitude. In string theories the differences between the bare Yukawa couplings are typically of $O(1)$ for the allowed couplings so it seems unlikely such bare threshold corrections will give a large change to the unificaion scale.

\section{Summary}

Motivated by the structure of many Grand Unified and Superstring theories, we have considered the implications of extending the structure of the MSSM by the addition of extra copies of massive vectorlike $(I+\bar{I})$ representations. These we take to fill out complete $S U(5)$ multiplets, even though there may be no stage of Grand Unification. While such representations do not affect the one loop unification predictions, they can have a substantial effect through their threshold effects and at the twoloop level. We computed their effects on the unification predictions for the running gauge couplings for the case the additional representations have common bare mass. We found that they always increase the value of $\alpha_{3}$ (ignoring Yukawa couplings effects) and thus, cannot explain the (marginal) discrepancy between the predicted value $\alpha_{3}\left(M_{Z}\right) \geq 0.125$ and the observed value $\alpha_{3}\left(M_{Z}\right)=0.118 \pm 0.003$.

We also found that the unification scale is systematically increased by the addition of such heavy states. The increase depends on the value of the coupling at unification and is largest for the case this is approaching the strong coupling limit. For this case the unification scale increases by a factor 
varying (function of $n$ ) between 1 and 5 for $\alpha_{g}=0.3$, at the limit of the perturbative analysis. The larger enhancement, allowing for the change in the string prediction at larger coupling, is only slightly closer to the value predicted in the (weakly coupled) heterotic string theory.

\section{Acknowledgments}

The authors want to thank G. Amelino-Camelia, K. Dienes, D.R.T. Jones, I. Kogan, J. March-Russell, M. Shifman for useful discussions. D.G. gratefully acknowledges the financial support from the part of University of Oxford and Oriel College (University of Oxford).

\section{Appendix}

The one loop beta function in the presence of complete $S U(5)$ multiplets is given by:

$$
b_{i}^{\prime}=\left(\begin{array}{r}
\frac{33}{5}+n \\
1+n \\
-3+n
\end{array}\right)
$$

where $n$ represents the linear combination $n=\left(n_{5}+3 n_{10}\right) / 2$, with $n_{5}=N_{\mathbf{5}}+N_{\overline{\mathbf{5}}}$ and $n_{10}=N_{\mathbf{1 0}}+N_{\overline{\mathbf{1 0}}}$. The one loop MSSM beta function is given by $b_{i}=b_{i}^{\prime}(n=0)$.

The two loop beta function in the presence of the complete $S U(5)$ multiplets is given by:

$$
b_{i j}^{\prime}=\left(\begin{array}{ccc}
\frac{199}{25}+\frac{7}{30} n_{5}+\frac{23}{10} n_{10} & \frac{27}{5}+\frac{9}{10} n_{5}+\frac{3}{10} n_{10} & \frac{88}{5}+\frac{16}{15} n_{5}+\frac{24}{5} n_{10} \\
\frac{9}{5}+\frac{3}{10} n_{5}+\frac{1}{10} n_{10} & 25+\frac{7}{2} n_{5}+\frac{21}{2} n_{10} & 24+8 n_{10} \\
\frac{11}{5}+\frac{2}{15} n_{5}+\frac{3}{5} n_{10} & 9+3 n_{10} & 14+\frac{17}{3} n_{5}+17 n_{10}
\end{array}\right)
$$

The MSSM two loop beta function is given by $b_{i j}=b_{i j}^{\prime}\left(n_{5}=0, n_{10}=0\right)$.

The values of the coefficients $\beta_{j, \psi}$ used in text, are given by the following matrix with the symmetry group index $\mathrm{j}$ as a line index $j=\{1,2,3\}$, and with the type of the field $\psi$ as a column index running over the set $\left\{l, d^{c}, e^{c}, u^{c}, q\right\}$, in this order.

$$
\beta_{j, \psi}=\left(\begin{array}{ccccc}
\frac{3}{10} & \frac{1}{5} & \frac{3}{5} & \frac{4}{5} & \frac{1}{10} \\
\frac{1}{2} & 0 & 0 & 0 & \frac{3}{2} \\
0 & \frac{1}{2} & 0 & \frac{1}{2} & 1
\end{array}\right)_{j, \psi}
$$

In the same notation we have for the matrix $\eta_{j, \psi}$

$$
\eta_{j, \psi}=\left(\begin{array}{ccccc}
\frac{3}{10} & \frac{2}{15} & \frac{6}{5} & \frac{8}{15} & \frac{1}{30} \\
\frac{3}{2} & 0 & 0 & 0 & \frac{3}{2} \\
0 & \frac{8}{3} & 0 & \frac{8}{3} & \frac{8}{3}
\end{array}\right)_{j, \psi} \equiv 2 C_{j}(\psi)
$$

where $C_{j}(\psi)$ is the quadratic Casimir operator, given by $3 Y_{\psi}^{2} / 5,3 / 4,4 / 3$ for $U(1), S U(2), S U(3)$ respectively.

With these definitions, we get,

$$
\sum_{\psi=\left\{l, d^{c}, e^{c}, u^{c}, q\right\}} N_{\psi} \beta_{i, \psi} \eta_{j, \psi}=\frac{1}{2}\left(b_{i j}^{\prime}-b_{i j}-2 n \lambda_{j} \delta_{i j}\right)
$$


with $\lambda_{1}=0, \lambda_{2}=2, \lambda_{3}=3$.

Throughout the text we used $S U(5)$ normalization for gauge couplings, $t=1 /(2 \pi) \ln \left(\right.$ scale $\left./ M_{g}\right)$, $\alpha_{i}=g_{i}^{2} /(4 \pi), Y_{\psi}=\lambda_{\psi}^{2} /(4 \pi)$.

In two loop, the equation for $\xi$ used in Section 4 is

$$
\alpha_{g}^{-1}-\alpha_{g}^{o-1}+n \xi=-\frac{1168}{231 \pi} \ln \left[\frac{\alpha_{g}}{\alpha_{g}^{o}}\right]+\sum_{j=1}^{3} \Delta_{j} \ln \left[1+b_{j}^{\prime} \alpha_{g} \xi\right]
$$

with $\Delta_{j}$ given by

$$
\Delta_{j}=\left\{\frac{13 n}{1320 b_{1}^{\prime} \pi}, \frac{291 n}{56 b_{2}^{\prime} \pi}, \frac{-24 n}{7 b_{3}^{\prime} \pi}\right\}
$$

The derivative of $\alpha_{3}\left(M_{z}\right)$ (eq.(32)), with respect to $z=\alpha_{g} / \alpha_{g}^{o}$ is positive, given that $z \geq 1$ and $n \geq 4$ as it can be seen from its expression given below:

$$
\begin{gathered}
\frac{\partial}{\partial z} \alpha_{3}\left(M_{z}\right)=3\left[320+240(-4+n)+60(-4+n)^{2}+5(-4+n)^{3}+2720(-1+z)\right. \\
+1600(-4+n)(-1+z)+290(-4+n)^{2}(-1+z)+15(-4+n)^{3}(-1+z) \\
+5716(-1+z)^{2}+2789(-4+n)(-1+z)^{2}+400(-4+n)^{2}(-1+z)^{2} \\
+15(-4+n)^{3}(-1+z)^{2}+496(-1+z)^{3}+1429(-4+n)(-1+z)^{3} \\
\left.+170(-4+n)^{2}(-1+z)^{3}+5(-4+n)^{3}(-1+z)^{3}\right] \times \\
\{14 \pi[n+5(-1+z)+(-4+n)(-1+z)] \times \\
{[20+5(-4+n)+53(-1+z)+5(-4+n)(-1+z)] \times} \\
z[-1+n+(-4+n)(-1+z)+z]\}^{-1} \alpha_{3}^{2}\left(M_{z}\right)
\end{gathered}
$$

\section{References}

[1] K.R. Dienes, A.E. Faraggi and J. March-Russell, Nucl. Phys. B467 (1996) 44.

[2] W.Pokorski, G.G.Ross, preprint OUTP-97-34-P(1997), hep-ph/9707402.

[3] M. Shifman, Mod.Phys.Lett.A10 (1995), 605 and references therein.

[4] J. Bagger, K. Matchev and D. Pierce, Phys.Lett.B348 (1995), 443;

P.H. Chankowski, Z. Pluciennik and S. Pokorski, Nucl.Phys.B439 (1995), 23;

M. Bastero-Gil and J. Perez-Mercader, Nucl.Phys.B450 (1995), 21.

[5] Review of Particle Data, Phys. Rev D54 (1996), 83.

[6] P. Ginsparg, Phys.Lett.B197 (1987), 139;

V.S. Kaplunovsky, Nucl.Phys.B307 (1988), 145;

Erratum: ibid. B382 (1992), 436.

[7] K.R. Dienes, Phys. Rept. 287 (1997) 447 and references therein.

[8] E. Witten, Nucl. Phys. B471 (1996) 135;

P.Horava, Phys. Rev. D 54 (1996), 7561. and

P.Horava, E. Witten, Nucl. Phys. B475 (1996), 94. 
[9] K.S. Babu and J.C. Pati, Phys.Lett.B384 (1996), 140

[10] R. Hempfling, Phys.Lett. B 351 (1995), 206

[11] M. Shifman, Int.J.Mod.Phys. A11 (1996) 5761, Talk at the International Workshop on Supersymmetry and Unification of Fundamental Interactions SUSY-96, University of Maryland, College Park, MD 20742, USA.

Other related works:

M.A. Shifman, A.I. Vainshtein, Nucl.Phys. B 277 (1986), 456.

M.A. Shifman, A. I. Vainshtein, Nucl. Phys. B 359 (1991), 571.

H. S. Li, K. T. Mahanthappa, Phys. Lett. B 319 (1993), 152.

M. Dine, Y. Shirman, Phys. Rev. D 50 (1994), 5389.

M. Shifman, preprint TPI-MINN-97/09-T, UMN-TH-1535-97, hep-ph/9704114

J. Hisano, M. Shifman, preprint TPI-MINN-97/14-T, UMN-TH-1543-97, hep-ph/9705417

N.Arkani-Hamed, H. Murayama, preprint LBNL-40346, UCB-PTH-97/21, hep-ph/9705189

[12] L.Ibáñez, G.G. Ross, Phys. Lett. 110 (1982) 215.

K. Inoue et al., Prog.Theor. Phys. 68 (1982) 927

L. Alvarez-Gaume, J. Polchinsky and M. Wise, Nucl. Phys. B 221(1983) 495.

[13] P.Langacker and N. Polonsky, Phys.Rev.D47 (1993), 4028.

[14] M.Carena, S.Pokorski and C.E.M.Wagner, Nucl.Phys.B406 (1993), 59;

P.Langacker and N. Polonsky, Phys.Rev.D47 (1993), 4028;

D.Pierce, hep-ph/9701344.

[15] I. Jack, D.R.T. Jones and C.G. North, Phys.Lett. B 386 (1996) 138.

[16] C. Kolda, J.March-Russell, hep-ph/9609480

[17] D. Ghilencea, M. Lanzagorta, G. Ross, preprint OUTP-97-32-P(1997), hep-ph/9707462. 


\section{Figure captions}

Figure 1. The ratio $M_{g} / M_{g}^{o}$ plotted in function of the ratio $\alpha_{g} / \alpha_{g}^{o}$ for different values of $n$.

Figure 2. The values of $\alpha_{3}\left(M_{z}\right)$ plotted in function of the ratio $\alpha_{g} / \alpha_{g}^{o}$ for different values of $n$.

Figure 3. The values of $\log _{10}\left(\mu_{e^{c}} / M_{g}^{o}\right)$ plotted in function of the ratio $\alpha_{g} / \alpha_{g}^{o}$ for different values of $n$.

Figure 4. The values of $\log _{10}\left(\mu_{q} / M_{g}^{o}\right)$ plotted in function of the ratio $\alpha_{g} / \alpha_{g}^{o}$ for different values of $n$.

Figure 5. The values of the splitting $\log _{10}\left(\mu_{q} / \mu_{e^{c}}\right)$ plotted in function of the ratio $\alpha_{g} / \alpha_{g}^{o}$ for different values of $n$. 

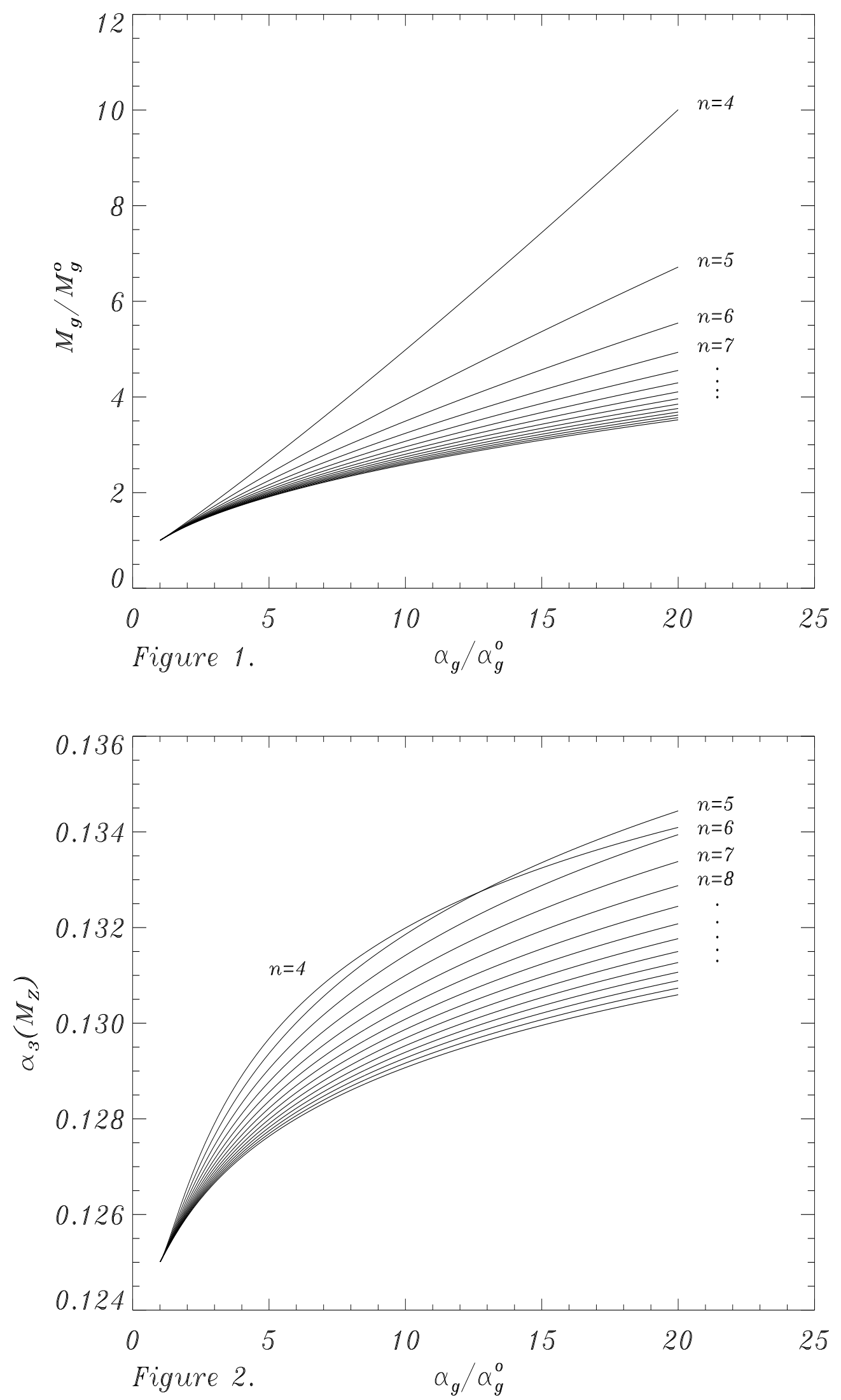

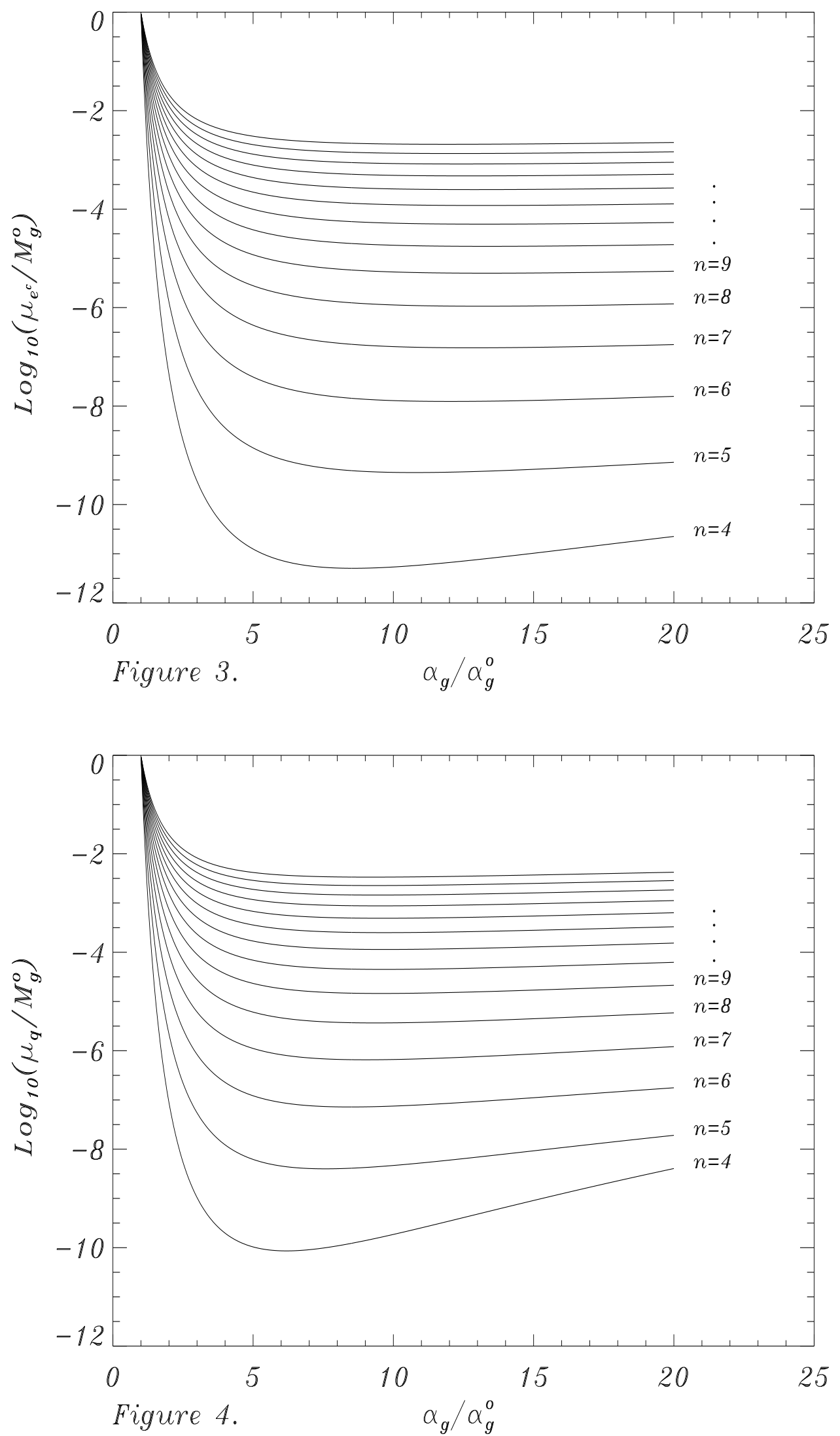


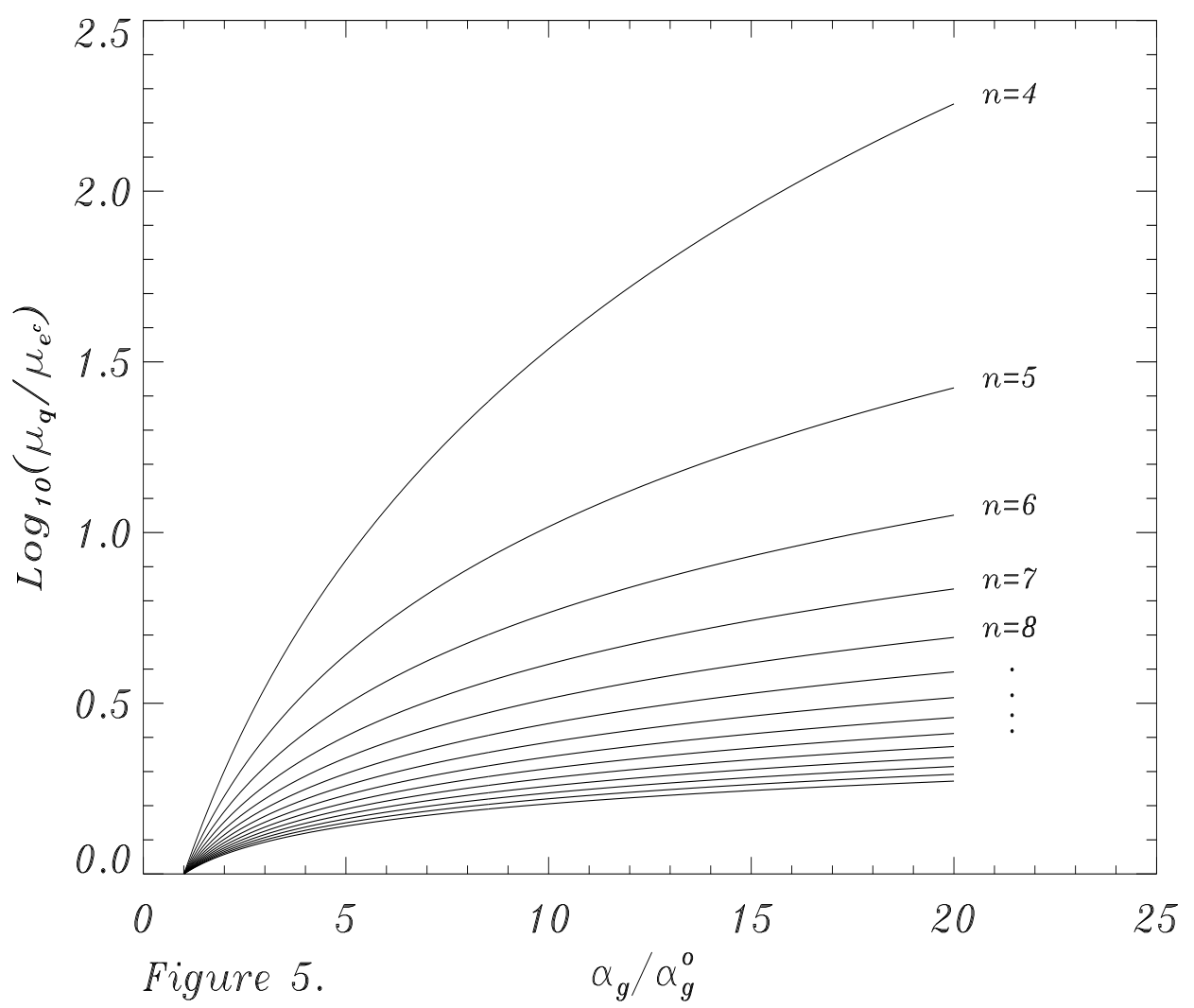

